\title{
CORRESPONDENCE
}

\section{Curb deep-sea mining now}

Cindy Lee Van Dover in her review of the Bismarck Sea mining project (Nature 470, $31-33$; 2011) accepts the inevitability of interest in excavating the sediments of hydrothermal vents for minerals such as copper, zinc, gold and silver. Many of the hundreds of these sites are accessible, and the issue is widely seen as not whether mining should proceed, but how it can be done profitably and safely.

I approach the issue with a strong bias, based on efforts over decades to figure out how to keep the world working as a biophysical system capable of serving indefinitely as a human habitat. On the overall issue I am not optimistic. On one topic, however, I am certain: the integrity of the oceanic biophysical system is being lost now and the human cost is overwhelming.

The fact is that intrusions into the global environment have passed a limit of acceptability and this one must be seen for the twofold attack on the global commons that it is.

Hydrothermal vents are one of the wonders of Earth: communities of autotrophic organisms that survive on Earth's energy as opposed to photosynthetic energy from the Sun, the source of energy of almost all other life. Each vent site may have its own high degree of endemism, essentially unique life. The mere fact that the sites are commercially attractive as ore is not an adequate reason to exploit them, any more than the existence of the giant redwoods of the Sierra Nevada justifies harvesting them for shingles. The vents are a window onto the history of life. By what right do we destroy them for corporate profit?

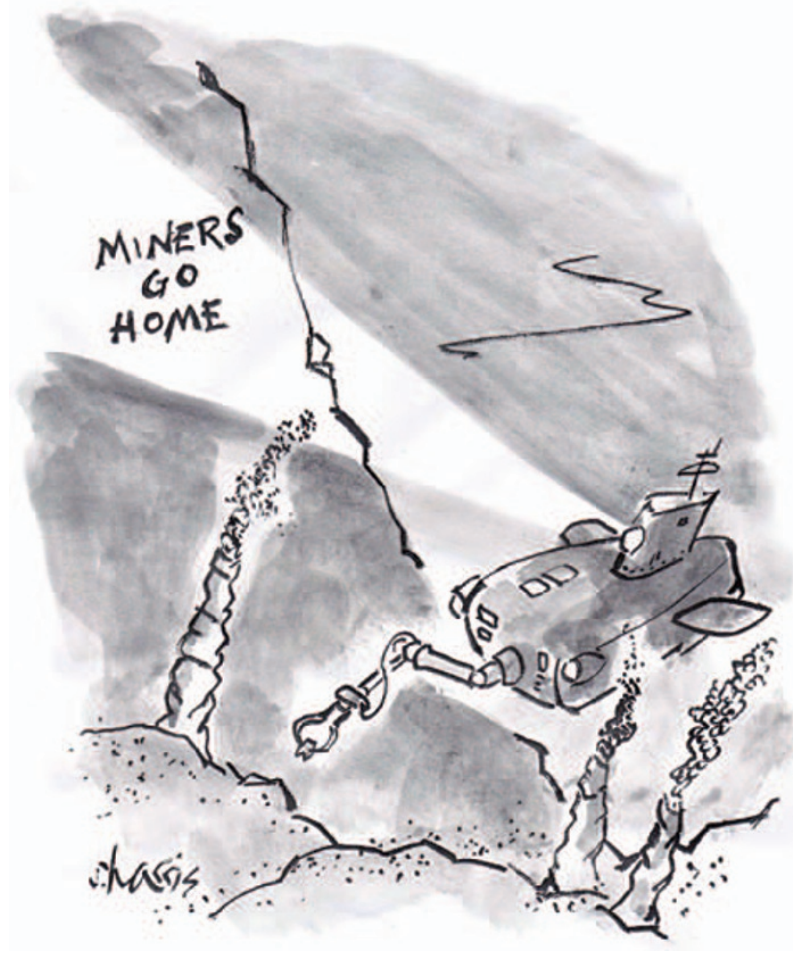

Worse, mining of marine sediments mobilizes the noxious minerals they contain, including those that are toxic to other marine life. Suddenly, we have another contribution to the chemical disruption of the ocean. We are well aware of the process and its effects. Do we need more research to confirm our experience?

Global problems all have local origins. Here we have the beginning of another process we shall never be able to stop, once started. Another mountain-top mining. Another Tar Sands of Alberta. Another North Slope oil development. Scientists who join the programme are offering tacit approval of it, no matter what their perspectives. The world is too small for this further destructive intrusion it should be stopped now before it becomes another corporate atrocity, too big and too valuable to stop.

George M. Woodwell Woods Hole Research Center, USA. gmwoodwell@whrc.org

\section{NIH plan will hinder translational studies}

The proposal by the US National Institutes of Health (NIH) to dismantle the National Center for Research Resources (NCRR) (see go.nature.com/yw3cq3) is more likely to inhibit than enhance translational research.

Through its Division of Comparative Medicine (DCM), the NCRR has long promoted translational research by supporting facilities and by providing resources and training to identify and target disease mechanisms. The proposed replacement for the NCRR, the National Center for Advancing Translational Sciences (NCATS), acknowledges the value of an integrated DCM by retaining its core functions as a cohesive programme within an 'Infrastructure Entity'.

However, in our view, the vision of NCATS as an incubator for innovative medicines is unrealistic. A major obstacle to developing new treatments through translational science is an inadequate understanding of basic biological pathways and mechanisms - not anaemic efforts by industry to test potential drug candidates. Using the NCRR's existing research resources as a means of enhancing the NIH's traditional strength in mechanistic research is a more certain route to translational success than focusing on chemical screening and intramural bioassays, as proposed for NCATS.

As veteran comparative biologists, we feel that the decision to slash the NCRR to initiate NCATS was undertaken without due diligence or sufficient opportunity for public debate. The rush to establish NCATS without a settled organizational plan and against the advice of numerous translational science researchers bodes ill for the new centre's ability to perform meaningful translational research in the foreseeable future.

The preservation of the DCM in the Infrastructure Entity will maintain core NIH translational science functions. The sprint to form NCATS by dismembering the NCRR might be good politics, but it is bad public policy.

Brad Bolon on behalf of 25 co-authors*, GEMpath,

Colorado, USA.

brad@gempath.net

${ }^{*}$ See http://dx.doi.org/10.1038/

$471036 b$ for a full list of signatories. SEENEWS P.I5

\section{Neuroscience cuts will hurt key areas}

We call on the UK Biotechnology and Biological Sciences Research Council (BBSRC) to reconsider its intention to cut funding for neuroscience by around $20 \%$ 